\title{
Integral Inequalities on Time Scales via the Theory of Isotonic Linear Functionals
}

\author{
Matloob Anwar, ${ }^{1}$ Rabia Bibi, ${ }^{1}$ \\ Martin Bohner, ${ }^{2}$ and Josip Pečarić ${ }^{3,4}$ \\ ${ }^{1}$ Centre for Advanced Mathematics and Physics, National University of Sciences and Technology, \\ Islamabad 44000, Pakistan \\ ${ }^{2}$ Department of Mathematics and Statistics, Missouri University of Science and Technology, Rolla, \\ MI 65409-0020, USA \\ ${ }^{3}$ Abdus Salam School of Mathematical Sciences, GC University, Lahore 54000, Pakistan \\ ${ }^{4}$ Faculty of Textile Technology, University of Zagreb, Pierottijeva 6, 10000 Zagreb, Croatia
}

Correspondence should be addressed to Matloob Anwar, matloob_t@yahoo.com

Received 26 April 2011; Accepted 23 May 2011

Academic Editor: Allan C. Peterson

Copyright (C) 2011 Matloob Anwar et al. This is an open access article distributed under the Creative Commons Attribution License, which permits unrestricted use, distribution, and reproduction in any medium, provided the original work is properly cited.

We apply the theory of isotonic linear functionals to derive a series of known inequalities, extensions of known inequalities, and new inequalities in the theory of dynamic equations on time scales.

\section{Introduction}

A time scale is an arbitrary nonempty closed subset of the real numbers. For an introduction to the theory of dynamic equations on time-scale, we refer to [1]. For functions defined on a time scale, we can consider the derivative and also the integral. For example, when the time scale is the set of all real numbers, the time-scale integral is an ordinary integral; when the time scale is the set of all integers, the time-scale integral is a sum; when the time scale is the set of all integer powers of a fixed number, the time-scale integral is a Jackson integral.

In this paper, we present a series of inequalities for the time-scale integral. Among the inequalities presented, we offer time-scale versions of Jensen's-inequality, Jensen-type inequalities, converses of Jensen's inequality, inequalities for means, Hölder's inequality, Minkowski's inequality, Dresher's inequality, Aczél's inequality, Popoviciu's inequality, and Diaz-Metcalf's inequality. 
The monograph [2] contains numerous classical inequalities that are proved for the so-called isotonic linear functionals. Since the time-scale integral is in fact an isotonic linear functional, the results from [2] can be applied to this setting. Our work shows that it is not necessary to prove such kinds of inequalities "from scratch" in the time-scale setting as they can all be obtained easily from well-known inequalities for isotonic linear functionals.

The setup of this paper is as follows. In the next section, we review some known results from the literature concerning Jensen's inequality on time-scale. Section 3 contains the definition of an isotonic linear functional and the confirmations that the time-scale Cauchy delta, Cauchy nabla, $\alpha$-diamond, multiple Riemann, and multiple Lebesgue integrals all are indeed isotonic linear functionals. Section 4 then is devoted to the time-scale Jensen's inequality and some of its generalizations. Some converses of Jensen's inequality in the form of time-scale Hermite-Hadamard's inequality and generalizations of it are contained in Section 5. Section 6 presents the multidimensional time-scale versions of Hölder's and Cauchy-Schwarz's inequality, followed in Section 7 by Minkowski's inequality. Section 8 is concerned with Dresher's inequality, and Section 9 offers time-scale versions of Aczél's and Popoviciu's inequalities. Section 10 contains Bellman's inequality and Section 11 deals with the Diaz-Metcalf inequality and some consequences. Five further converses of Jensen's inequality are contained in the final Section 12.

\section{Known Results Concerning Jensen's Inequality}

Jensen's inequality is of great interest in the theories of differential and difference equations as well as other areas of mathematics. The original Jensen inequality can be stated as follows.

Theorem 2.1 (Jensen's inequality [3, Formula (5')]). Let $a, b \in \mathbb{R}$ with $a<b$, and suppose $I \subset \mathbb{R}$ is an interval. If $\Phi \in C(I, \mathbb{R})$ is convex and $f \in C([a, b], I)$, then

$$
\Phi\left(\frac{\int_{a}^{b} f(t) \mathrm{d} t}{b-a}\right) \leq \frac{\int_{a}^{b} \Phi(f(t)) \mathrm{d} t}{b-a} .
$$

The Jensen inequality on time-scale has been obtained by Agarwal et al. [4].

Theorem 2.2 (Jensen's inequality [1, Theorem 6.17]). Let $a, b \in \mathbb{T}$ with $a<b$, and suppose $I \subset \mathbb{R}$ is an interval. If $\Phi \in C(I, \mathbb{R})$ is convex and $f \in C_{\mathrm{rd}}([a, b], I)$, then

$$
\Phi\left(\frac{\int_{a}^{b} f(t) \Delta t}{b-a}\right) \leq \frac{\int_{a}^{b} \Phi(f(t)) \Delta t}{b-a}
$$

When $\mathbb{T}=\mathbb{R}$ in Theorem 2.2, we obtain Theorem 2.1. When $\mathbb{T}=\mathbb{Z}$ in Theorem 2.2, we get the usual geometric-arithmetic mean inequality.

The following result is given by Wong et al. in [5]. When $h(t) \equiv 1$ in Theorem 2.3 , we obtain Theorem 2.2. 
Theorem 2.3 (Jensen's inequality [5, Theorem 2.2]). Let $a, b \in \mathbb{T}$ with $a<b$, and suppose $I \subset \mathbb{R}$ is an interval. Assume $h \in C_{\mathrm{rd}}([a, b], \mathbb{R})$ satisfies $\int_{a}^{b}|h(t)| \Delta t>0$. If $\Phi \in C(I, \mathbb{R})$ is convex and $f \in C_{\mathrm{rd}}([a, b], I)$, then

$$
\Phi\left(\frac{\int_{a}^{b}|h(t)| f(t) \Delta t}{\int_{a}^{b}|h(t)| \Delta t}\right) \leq \frac{\int_{a}^{b}|h(t)| \Phi(f(t)) \Delta t}{\int_{a}^{b}|h(t)| \Delta t} .
$$

In [6], Özkan et al. proved that Theorem 2.3 is also true if we use the nabla integral (see [1, Section 8.4]) instead of the delta integral. In [7], Sheng et al. introduced the so-called $\alpha$-diamond integral, where $0 \leq \alpha \leq 1$. It is a linear combination of the delta integral and the nabla integral. When $\alpha=1$, we get the usual delta integral, and when $\alpha=0$, we get the usual nabla integral. The following result concerning the $\alpha$-diamond integral is given by Ammi et al. in [8] (see also [6]).

Theorem 2.4 (Jensen's inequality [8, Theorem 3.3]). Let $\alpha \in[0,1]$. Let $a, b \in \mathbb{T}$ with $a<b$ and suppose $I \subset \mathbb{R}$ is an interval. Assume $h \in C([a, b], \mathbb{R})$ satisfies $\int_{a}^{b}|h(t)| \nabla_{\alpha} t>0$. If $\Phi \in C(I, \mathbb{R})$ is convex and $f \in C([a, b], I)$, then

$$
\Phi\left(\frac{\int_{a}^{b}|h(t)| f(t) \diamond_{\alpha} t}{\int_{a}^{b}|h(t)| \diamond_{\alpha} t}\right) \leq \frac{\int_{a}^{b}|h(t)| \Phi(f(t)) \diamond_{\alpha} t}{\int_{a}^{b}|h(t)| \diamond_{\alpha} t} .
$$

\section{Isotonic Linear Functionals and Time-Scale Integrals}

We recall the following definition from [2, page 47].

Definition 3.1 (Isotonic linear functional). Let $E$ be a nonempty set and $L$ be a linear class of real-valued functions $f: E \rightarrow \mathbb{R}$ having the following properties:

$\left(L_{1}\right)$ If $f, g \in L$ and $a, b \in \mathbb{R}$, then $(a f+b g) \in L$.

$\left(L_{2}\right)$ If $f(t)=1$ for all $t \in E$, then $f \in L$.

An isotonic linear functional is a functional $A: L \rightarrow \mathbb{R}$ having the following properties:

$\left(A_{1}\right)$ If $f, g \in L$ and $a, b \in \mathbb{R}$, then $A(a f+b g)=a A(f)+b A(g)$.

$\left(A_{2}\right)$ If $f \in L$ and $f(t) \geq 0$ for all $t \in E$, then $A(f) \geq 0$.

When we use the approach of isotonic linear functionals as given in Definition 3.1, it is not necessary to know many details from the calculus of dynamic equations on time-scale. We only need to know that the time-scale integral is such an isotonic linear functional.

Theorem 3.2. Let $\mathbb{T}$ be a time scale. For $a, b \in \mathbb{T}$ with $a<b$, let

$$
E=[a, b) \cap \mathbb{T}, \quad L=C_{\mathrm{rd}}([a, b), \mathbb{R}) .
$$


Then $\left(L_{1}\right)$ and $\left(L_{2}\right)$ are satisfied. Moreover, let

$$
A(f)=\int_{a}^{b} f(t) \Delta t
$$

where the integral is the Cauchy delta time-scale integral. Then $\left(A_{1}\right)$ and $\left(A_{2}\right)$ are satisfied.

Proof. This follows from [1, Definition 1.58 and Theorem 1.77].

Instead of recalling the formal definition of the time-scale integral and the definition of the set of rd-continuous functions $C_{r d}$ used in Theorem 3.2, which can be found in [1, Section 1.4], we choose to only give a few examples.

Example 3.3. If $\mathbb{T}=\mathbb{R}$ in Theorem 3.2, then $L=C([a, b], \mathbb{R})$ and

$$
A(f)=\int_{a}^{b} f(t) \mathrm{d} t
$$

If $\mathbb{T}=\mathbb{Z}$ in Theorem 3.2, then $L$ consists of all real-valued functions defined on $[a, b-1] \cap \mathbb{Z}$ and

$$
A(f)=\sum_{t=a}^{b-1} f(t)
$$

Let $h>0$. If $\mathbb{T}=h \mathbb{Z}$ in Theorem 3.2, then $L$ consists of all real-valued functions defined on $[a, b-h] \cap h \mathbb{Z}$ and

$$
A(f)=h \sum_{k=a / h}^{b / h-1} f(k)
$$

Let $q>1$. If $\mathbb{T}=q^{\mathbb{N}_{0}}$ in Theorem 3.2, then $L$ consists of all real-valued functions defined on $[a, b / q] \cap q^{\mathbb{N}_{0}}$ and

$$
A(f)=(q-1) \sum_{k=\log _{q}(a)}^{\log _{q}(b)-1} q^{k} f\left(q^{k}\right)
$$

Note that Theorem 3.2 also has corresponding versions for the nabla and $\alpha$-diamond integral, which are given next for completeness.

Theorem 3.4. Let $\mathbb{T}$ be a time scale. For $a, b \in \mathbb{T}$ with $a<b$, let

$$
E=(a, b] \cap \mathbb{T}, \quad L=C_{\mathrm{ld}}((a, b], \mathbb{R}) .
$$


Then $\left(L_{1}\right)$ and $\left(L_{2}\right)$ are satisfied. Moreover, let

$$
A(f)=\int_{a}^{b} f(t) \nabla t
$$

where the integral is the Cauchy nabla time-scale integral. Then $\left(A_{1}\right)$ and $\left(A_{2}\right)$ are satisfied.

Proof. This follows from [1, Definition 8.43 and Theorem 8.47].

Theorem 3.5. Let $\mathbb{T}$ be a time scale. For $a, b \in \mathbb{T}$ with $a<b$, let

$$
E=[a, b] \cap \mathbb{T}, \quad L=\mathrm{C}([a, b], \mathbb{R}) .
$$

Then $\left(L_{1}\right)$ and $\left(L_{2}\right)$ are satisfied. Moreover, let

$$
A(f)=\int_{a}^{b} f(t) \diamond_{\alpha} t
$$

where the integral is the Cauchy $\alpha$-diamond time-scale integral. Then $\left(A_{1}\right)$ and $\left(A_{2}\right)$ are satisfied.

Proof. This follows from [7, Definition 3.2 and Theorem 3.7].

Multiple Riemann integration on time-scale was introduced in [9]. The Riemann integral introduced there is also an isotonic linear functional.

Theorem 3.6. Let $\mathbb{T}_{1}, \ldots, \mathbb{T}_{n}$ be time-scale. For $a_{i}, b_{i} \in \mathbb{T}_{i}$ with $a_{i}<b_{i}, 1 \leq i \leq n$, let

$$
E \subset\left(\left[a_{1}, b_{1}\right) \cap \mathbb{T}_{1}\right) \times \cdots \times\left(\left[a_{n}, b_{n}\right) \cap \mathbb{T}_{n}\right)
$$

be Jordan $\Delta$-measurable and let $L$ be the set of all bounded $\Delta$-integrable functions from $E$ to $\mathbb{R}$. Then $\left(L_{1}\right)$ and $\left(L_{2}\right)$ are satisfied. Moreover, let

$$
A(f)=\int_{E} f(t) \Delta t
$$

where the integral is the multiple Riemann delta time-scale integral. Then $\left(A_{1}\right)$ and $\left(A_{2}\right)$ are satisfied.

Proof. This follows from [9, Definition 4.13 and Theorem 3.4].

From [9, Remark 2.18], it is also clear that a theorem similar to Theorem 3.6 is also true for the nabla case or any mixture of delta and nabla integrals in the multiple variable case.

The multiple Lebesgue integration on time-scale was introduced in [10]. The Lebesgue integral introduced there is also an isotonic linear functional.

Theorem 3.7. Let $\mathbb{T}_{1}, \ldots, \mathbb{T}_{n}$ be time-scale. For $a_{i}, b_{i} \in \mathbb{T}_{i}$ with $a_{i}<b_{i}, 1 \leq i \leq n$, let

$$
E \subset\left(\left[a_{1}, b_{1}\right) \cap \mathbb{T}_{1}\right) \times \cdots \times\left(\left[a_{n}, b_{n}\right) \cap \mathbb{T}_{n}\right)
$$


be Lebesgue $\Delta$-measurable and let $L$ be the set of all $\Delta$-measurable functions from $E$ to $\mathbb{R}$. Then $\left(L_{1}\right)$ and $\left(L_{2}\right)$ are satisfied. Moreover, let

$$
A(f)=\int_{E} f(t) \Delta t
$$

where the integral is the multiple Lebesgue delta time-scale integral. Then $\left(A_{1}\right)$ and $\left(A_{2}\right)$ are satisfied. Proof. This follows from [10, Section 3].

Theorem 3.8. Under the assumptions of Theorem 3.7, let $A(f)$ be replaced by

$$
A(f)=\frac{\int_{E}|h(t)| f(t) \Delta t}{\int_{E}|h(t)| \Delta t}
$$

where $h: E \rightarrow \mathbb{R}$ is $\Delta$-integrable such that $\int_{E}|h(t)| \Delta t>0$. Then $A$ is an isotonic linear functional satisfying $A(1)=1$.

\section{Jensen's Inequality}

Jessen in [11] gave the following generalization of Jensen's inequality for isotonic linear functionals.

Theorem 4.1 (Jessen's inequality [2, Theorem 2.4]). Let $L$ satisfy properties $\left(L_{1}\right)$ and $\left(L_{2}\right)$. Assume $\Phi \in C(I, \mathbb{R})$ is convex, where $I \subset \mathbb{R}$ is an interval. If $A$ satisfies $\left(A_{1}\right)$ and $\left(A_{2}\right)$ such that $A(1)=1$, then for all $f \in L$ such that $\Phi(f) \in L$, one has $A(f) \in I$ and

$$
\Phi(A(f)) \leq A(\Phi(f))
$$

Now our first result is the following generalization of Jensen's inequality.

Theorem 4.2 (Jensen's inequality). Assume $\Phi \in \mathrm{C}(I, \mathbb{R})$ is convex, where $I \subset \mathbb{R}$ is an interval. Let $E \subset \mathbb{R}^{n}$ be as in Theorem 3.7 and suppose $f$ is $\Delta$-integrable on $E$ such that $f(E)=I$. Moreover, let $h: E \rightarrow \mathbb{R}$ be $\Delta$-integrable such that $\int_{E}|h(t)| \Delta t>0$. Then

$$
\Phi\left(\frac{\int_{E}|h(t)| f(t) \Delta t}{\int_{E}|h(t)| \Delta t}\right) \leq \frac{\int_{E}|h(t)| \Phi(f(t)) \Delta t}{\int_{E}|h(t)| \Delta t} .
$$

Proof. Just apply Theorems 4.1 and 3.8.

Remark 4.3 (Jensen's inequality). Note that the known results from Section 2 follow from Theorem 4.1 in the same way as Theorem 4.2 does: Theorem 2.3 follows as in Theorems 3.2 and 2.4 follows as in Theorem 3.5. Note also that a similar theorem for the multiple Riemann integral can be stated and proved using Theorem 3.6. This will be the case for all inequalities stated in this paper; however, we only explicitly state each time the case for the multiple Lebesgue integral. 


\section{Hermite-Hadamard's Inequality}

Beesack and Pečarić in [12] gave the following generalization of Hermite-Hadamard's inequality for isotonic linear functionals.

Theorem 5.1 (Beesack-Pečarić's inequality [2, Theorem 3.37]). Let L satisfy properties $\left(L_{1}\right)$ and $\left(L_{2}\right)$. Assume $\Phi \in \mathrm{C}(I, \mathbb{R})$ is convex, where $I=[m, M] \subset \mathbb{R}$ with $m<M$. If A satisfies $\left(A_{1}\right)$ and $\left(A_{2}\right)$ such that $A(1)=1$, then for all $f \in L$ such that $\Phi(f) \in L$, one has

$$
A(\Phi(f)) \leq \frac{M-A(f)}{M-m} \Phi(m)+\frac{A(f)-m}{M-m} \Phi(M)
$$

Theorem 5.2 (Hermite-Hadamard's inequality). Assume $\Phi \in \mathrm{C}(I, \mathbb{R})$ is convex, where $I=$ $[m, M] \subset \mathbb{R}$ with $m<M$. Let $E \subset \mathbb{R}^{n}$ be as in Theorem 3.7, and suppose $f$ is $\Delta$-integrable on $E$ such that $f(E)=I$. Moreover, let $h: E \rightarrow \mathbb{R}$ be $\Delta$-integrable such that $\int_{E}|h(t)| \Delta t>0$. Then

$$
\begin{aligned}
\frac{\int_{E}|h(t)| \Phi(f(t)) \Delta t}{\int_{E}|h(t)| \Delta t} \leq & \frac{M-\int_{E}|h(t)| f(t) \Delta t / \int_{E}|h(t)| \Delta t}{M-m} \Phi(m) \\
& +\frac{\int_{E}|h(t)| f(t) \Delta t / \int_{E}|h(t)| \Delta t-m}{M-m} \Phi(M) .
\end{aligned}
$$

Proof. Just apply Theorems 5.1 and 3.8.

Remark 5.3 (Hermite-Hadamard's inequality). Note that the known result [13, Theorem 3.14] (see also $[14,15]$ ) follows from Theorem 5.1 in the same way as Theorem 5.2 does, this time applying Theorem 3.5 .

A combination of Theorem 4.1 and Theorem 5.1 in a slightly different form is given by Pečarić and Beesack in [16] as follows.

Theorem 5.4 (Pečarić-Beesack's inequality [2, Theorem 5.13]). Let L satisfy properties $\left(L_{1}\right)$ and $\left(L_{2}\right)$. Assume $\Phi \in C(I, \mathbb{R})$ is convex, where $[m, M] \subset I$ with $m<M$ and $I \subset \mathbb{R}$ is an interval. Suppose A satisfies $\left(A_{1}\right)$ and $\left(A_{2}\right)$ such that $A(1)=1$. Let $f \in L$ such that $f(E) \subset[m, M]$ and $\Phi(f) \in L$, and define $p, q \geq 0$ such that $p+q>0$ and

$$
A(f)=\frac{p m+q M}{p+q}
$$

holds. Then

$$
\Phi\left(\frac{p m+q M}{p+q}\right) \leq A(\Phi(f)) \leq \frac{p \Phi(m)+q \Phi(M)}{p+q}
$$

Theorem 5.5 (Hermite-Hadamard's inequality). Assume $\Phi \in C(I, \mathbb{R})$ is convex, where $[m, M] \subset I$ with $m<M$ and $I \subset \mathbb{R}$ is an interval. Let $E \subset \mathbb{R}^{n}$ be as in Theorem 3.7 and suppose $f$ is 
$\Delta$-integrable on $E$ such that $f(E) \subset[m, M]$. Moreover, let $h: E \rightarrow \mathbb{R}$ be $\Delta$-integrable such that $\int_{E}|h(t)| \Delta t>0$. Let $p, q \geq 0$ be such that $p+q>0$ and

$$
\frac{\int_{E}|h(t)| f(t) \Delta t}{\int_{E}|h(t)| \Delta t}=\frac{p m+q M}{p+q}
$$

holds. Then

$$
\Phi\left(\frac{p m+q M}{p+q}\right) \leq \frac{\int_{E}|h(t)| \Phi(f(t)) \Delta t}{\int_{E}|h(t)| \Delta t} \leq \frac{p \Phi(m)+q \Phi(M)}{p+q} .
$$

Proof. Just apply Theorems 5.4 and 3.8.

\section{Hölder's Inequality}

We first recall Hölder's inequality for isotonic linear functionals as given in [2].

Theorem 6.1 (Hölder's inequality [2, Theorem 4.12]). Let $E, L$, and $A$ be such that $\left(L_{1}\right),\left(L_{2}\right)$, $\left(A_{1}\right)$, and $\left(A_{2}\right)$ are satisfied. For $p \neq 1$, define $q=p /(p-1)$. Assume $|w||f|^{p},|w||g|^{q},|w f g| \in L$. If $p>1$, then

$$
A(|w f g|) \leq A^{1 / p}\left(|w||f|^{p}\right) A^{1 / q}\left(|w||g|^{q}\right)
$$

This inequality is reversed if $0<p<1$ and $A\left(|w||g|^{q}\right)>0$, and it is also reversed if $p<0$ and $A\left(|w||f|^{p}\right)>0$.

Theorem 6.2 (Hölder's inequality). For $p>1$, define $q=p /(p-1)$. Let $E \subset \mathbb{R}^{n}$ be as in Theorem 3.7. Assume $|w||f|^{p},|w||g|^{q},|w f g|$ are $\Delta$-integrable on E. If $p>1$, then

$$
\int_{E}|w(t) f(t) g(t)| \Delta t \leq\left(\int_{E}|w(t)||f(t)|^{p} \Delta t\right)^{1 / p}\left(\int_{E}|w(t)||g(t)|^{q} \Delta t\right)^{1 / q} .
$$

This inequality is reversed if $0<p<1$ and $\int_{E}|w(t)||g(t)|^{q} \Delta t>0$, and it is also reversed if $p<0$ and $\int_{E}|w(t)||f(t)|^{p} \Delta t>0$.

Proof. Just apply Theorems 6.1 and 3.7.

Remark 6.3 (Hölder's inequality). Note that the known results from the time-scale literature follow from Theorem 6.1 in the same way as Theorem 6.2 does: [1, Theorem 6.13] follows as in Theorem 3.2 and $[8$, Theorem 4.1] (see also $[17,18]$ ) follows as in Theorem 3.5.

Theorem 6.4 (Cauchy-Schwarz's inequality). Let $E \subset \mathbb{R}^{n}$ be as in Theorem 3.7. If $|w| f^{2},|w| g^{2}$, $|w f g|$ are $\Delta$-integrable on $E$, then

$$
\int_{E}|w(t) f(t) g(t)| \Delta t \leq \sqrt{\left(\int_{E}|w(t)| f^{2}(t) \Delta t\right)\left(\int_{E}|w(t)| g^{2}(t) \Delta t\right)} .
$$

Proof. Just let $p=2$ in Theorem 6.2. 


\section{Minkowski's Inequality}

Another classical inequality is Minkowski's inequality. We first recall Minkowski's inequality for isotonic linear functionals as given in [2].

Theorem 7.1 (Minkowski's inequality [2, Theorem 4.13]). Let $E, L$, and $A$ be such that $\left(L_{1}\right)$, $\left(L_{2}\right),\left(A_{1}\right)$, and $\left(A_{2}\right)$ are satisfied. For $p \in \mathbb{R}$, assume $|w||f|^{p},|w||g|^{p},|w||f+g|^{p} \in L$. If $p>1$, then

$$
A^{1 / p}\left(|w||f+g|^{p}\right) \leq A^{1 / p}\left(|w||f|^{p}\right)+A^{1 / p}\left(|w||g|^{p}\right) .
$$

This inequality is reversed if $0<p<1$ or $p<0$ provided $A\left(|w||f|^{p}\right)>0$ and $A\left(|w||g|^{p}\right)>0$ hold.

Theorem 7.2 (Minkowski's inequality). Let $E \subset \mathbb{R}^{n}$ be as in Theorem 3.7. For $p \in \mathbb{R}$, assume $|w||f|^{p},|w||g|^{p},|w||f+g|^{p}$ are $\Delta$-integrable on $E$. If $p>1$, then

$$
\begin{aligned}
\left(\int_{E}|w(t)||f(t)+g(t)|^{p} \Delta t\right)^{1 / p} \leq & \left(\int_{E}|w(t)||f(t)|^{p} \Delta t\right)^{1 / p} \\
& +\left(\int_{E}|w(t)||g(t)|^{p} \Delta t\right)^{1 / p} .
\end{aligned}
$$

This inequality is reversed for $0<p<1$ or $p<0$ provided each of the two terms on the right-hand side is positive.

Proof. Just apply Theorems 7.1 and 3.7.

Remark 7.3 (Minkowski's inequality). Note that the known results from the time-scale literature follow from Theorem 7.1 in the same way as Theorem 7.2 does: [1, Theorem 6.16] follows as in Theorem 3.2 and $[8$, Theorem 4.4] (see also $[17,18]$ ) follows as in Theorem 3.5.

\section{Dresher's Inequality}

If $n=2$ in the result of this section, then one has the so-called Dresher inequality (see [19, Section 7]). We first present the generalization of this inequality for isotonic linear functionals as given in [2].

Theorem 8.1 (Dresher's inequality [2, Theorem 4.21]). Let $E$ and $L$ be such that $\left(L_{1}\right),\left(L_{2}\right)$ are satisfied, and suppose that both $A$ and $B$ satisfy $\left(A_{1}\right),\left(A_{2}\right)$. If

$$
|w|\left|f_{i}\right|^{p}, \quad|w|\left(\sum_{i=1}^{n}\left|f_{i}\right|\right)^{p}, \quad|w|\left|g_{i}\right|^{r}, \quad|w|\left(\sum_{i=1}^{n}\left|g_{i}\right|\right)^{r} \in L
$$

where $p \geq 1>r>0$ and $B\left(|w|\left|g_{i}\right|^{r}\right)>0$ for $1 \leq i \leq n$, then

$$
\left(\frac{A\left(|w|\left(\sum_{i=1}^{n}\left|f_{i}\right|\right)^{p}\right)}{B\left(|w|\left(\sum_{i=1}^{n}\left|g_{i}\right|\right)^{r}\right)}\right)^{1 /(p-r)} \leq \sum_{i=1}^{n}\left(\frac{A\left(|w|\left|f_{i}\right|^{p}\right)}{B\left(|w|\left|g_{i}\right|^{r}\right)}\right)^{1 /(p-r)}
$$


Theorem 8.2 (Dresher's inequality). Let $E \subset \mathbb{R}^{n}$ be as in Theorem 3.7. If

$$
|w|\left|f_{i}\right|^{p}, \quad|w|\left(\sum_{i=1}^{n}\left|f_{i}\right|\right)^{p}, \quad|w|\left|g_{i}\right|^{r}, \quad|w|\left(\sum_{i=1}^{n}\left|g_{i}\right|\right)^{r}
$$

are $\Delta$-integrable on $E$, where $p \geq 1>r>0$ and $\int_{E}\left|w(t) \| g_{i}(t)\right|^{r} \Delta t>0$ for $1 \leq i \leq n$, then

$$
\left(\frac{\int_{E}|w(t)|\left(\sum_{i=1}^{n}\left|f_{i}(t)\right|\right)^{p} \Delta t}{\int_{E}|w(t)|\left(\sum_{i=1}^{n}\left|g_{i}(t)\right|\right)^{r} \Delta t}\right)^{1 /(p-r)} \leq \sum_{i=1}^{n}\left(\frac{\int_{E}|w(t)|\left|f_{i}(t)\right|^{p} \Delta t}{\int_{E}|w(t)|\left|g_{i}(t)\right|^{r} \Delta t}\right)^{1 /(p-r)} .
$$

Proof. Just apply Theorems 8.1 and 3.7.

Remark 8.3 (Dresher's inequality). Dresher's inequality on time-scale is new even for the cases of a single-variable Cauchy delta and nabla integral and also for the $\alpha$-diamond integral.

\section{Popoviciu's Inequality}

We first recall Popoviciu's inequality for isotonic linear functionals as given in [2].

Theorem 9.1 (Popoviciu's inequality [2, Theorem 4.27]). Let $E$, $L$, and $A$ be such that $\left(L_{1}\right),\left(L_{2}\right)$, $\left(A_{1}\right)$, and $\left(A_{2}\right)$ are satisfied. For $p \neq 1$, define $q=p /(p-1)$. Assume $|f|^{p},|g|^{q},|f g| \in L$. Suppose $f_{0}, g_{0}>0$ are such that

$$
f_{0}^{p}-A\left(|f|^{p}\right)>0, \quad g_{0}^{q}-A\left(|g|^{q}\right)>0
$$

If $p>1$, then

$$
\left(f_{0}^{p}-A\left(|f|^{p}\right)\right)^{1 / p}\left(g_{0}^{q}-A\left(|g|^{q}\right)\right)^{1 / q} \leq f_{0} g_{0}-A(|f g|) .
$$

This inequality is reversed if $0<p<1$ and $A\left(|g|^{q}\right)>0$ or if $p<0$ and $A\left(|f|^{p}\right)>0$.

Theorem 9.2 (Popoviciu's inequality). Let $E \subset \mathbb{R}^{n}$ be as in Theorem 3.7. For $p \neq 1$, define $q=$ $p /(p-1)$. Assume $|f|^{p},|g|^{q},|f g|$ are $\Delta$-integrable on E. Suppose $f_{0}, g_{0}>0$ are such that

$$
f_{0}^{p}-\int_{E}|f(t)|^{p} \Delta t>0, \quad g_{0}^{q}-\int_{E}|g(t)|^{q} \Delta t>0 .
$$

If $p>1$, then

$$
f_{0} g_{0}-\int_{E}|f(t) g(t)| \Delta t \geq\left(f_{0}^{p}-\int_{E}|f(t)|^{p} \Delta t\right)^{1 / p}\left(g_{0}^{q}-\int_{E}|g(t)|^{q} \Delta t\right)^{1 / q} .
$$

This inequality is reversed if $0<p<1$ and $\int_{E}|g(t)|^{q} \Delta t>0$ or if $p<0$ and $\int_{E}|f(t)|^{p} \Delta t>0$.

Proof. Just apply Theorems 8.1 and 3.7 . 
Theorem 9.3 (Aczél's inequality). Let $E \subset \mathbb{R}^{n}$ be as in Theorem 3.7. Assume $f^{2}, g^{2},|f g|$ are $\Delta$ integrable on $E$. If $f_{0}, g_{0}>0$ are such that

$$
f_{0}^{2}-\int_{E} f^{2}(t) \Delta t>0, \quad g_{0}^{2}-\int_{E} g^{2}(t) \Delta t>0,
$$

then

$$
f_{0} g_{0}-\int_{E}|f(t) g(t)| \Delta t \geq \sqrt{\left(f_{0}^{2}-\int_{E} f^{2}(t) \Delta t\right)\left(g_{0}^{2}-\int_{E} g^{2}(t) \Delta t\right)} .
$$

Proof. Just let $p=2$ in Theorem 9.2.

Remark 9.4 (Aczél's and Popoviciu's inequalities). Aczél's and Popoviciu's inequalities on time-scale are new even for the cases of a single-variable Cauchy delta and nabla integral and also for the $\alpha$-diamond integral. The original Aczél inequality can be found in [20]. For a version of Aczél's inequality for isotonic linear functionals, we refer to [2, Theorem 4.26].

\section{Bellman's Inequality}

We first recall Bellman's inequality for isotonic linear functionals as given in [2].

Theorem 10.1 (Bellman's inequality [2, Theorem 4.29]). Let $E, L$, and $A$ be such that $\left(L_{1}\right),\left(L_{2}\right)$, $\left(A_{1}\right)$, and $\left(A_{2}\right)$ are satisfied. For $p \in \mathbb{R}$, assume $|f|^{p},|g|^{p},(|f|+|g|) \in$ L. Suppose $f_{0}, g_{0}>0$ are such that

$$
f_{0}^{p}-A\left(|f|^{p}\right)>0, \quad g_{0}^{p}-A\left(|g|^{p}\right)>0
$$

If $p>1$, then

$$
\left(\left(f_{0}^{p}-A\left(|f|^{p}\right)\right)^{1 / p}+\left(g_{0}^{p}-A\left(|g|^{p}\right)\right)^{1 / p}\right)^{p} \leq\left(f_{0}+g_{0}\right)^{p}-A\left((|f|+|g|)^{p}\right) .
$$

This inequality is reversed if $0<p<1$ or $p<0$ and $A\left(|f|^{p}\right)>0$.

Theorem 10.2 (Bellman's inequality). Let $E \subset \mathbb{R}^{n}$ be as in Theorem 3.7. For $p \in \mathbb{R}$, assume $|f|^{p},|g|^{p},(|f|+|g|)$ are $\Delta$-integrable on E. Suppose $f_{0}, g_{0}>0$ are such that

$$
f_{0}^{p}-\int_{E}|f(t)|^{p} \Delta t>0, \quad g_{0}^{p}-\int_{E}|g(t)|^{p} \Delta t>0 .
$$


If $p>1$, then

$$
\left(\left(f_{0}^{p}-\int_{E}|f(t)|^{p} \Delta t\right)^{1 / p}+\left(g_{0}^{p}-\int_{E}|g(t)|^{p} \Delta t\right)^{1 / p}\right)^{p} \leq\left(f_{0}+g_{0}\right)^{p}-\int_{E}(|f(t)|+|g(t)|)^{p} \Delta t .
$$

This inequality is reversed if $0<p<1$ or $p<0$ and $\int_{E}|f(t)|^{p} \Delta t>0$.

Proof. Just apply Theorems 10.1 and 3.7.

\section{Diaz-Metcalf's Inequality}

If $p=q=2$ and $w=1$ in the first result of this section, then one has the so-called Diaz-Metcalf inequality. We first present the generalization of this inequality for isotonic linear functionals as given in [2].

Theorem 11.1 (Diaz-Metcalf's inequality [2, Theorem 4.14]). Let $E, L$, and $A$ be such that $\left(L_{1}\right)$, $\left(L_{2}\right),\left(A_{1}\right)$, and $\left(A_{2}\right)$ are satisfied. For $p \neq 1$, let $q=p /(p-1)$. Assume $|w||f|^{p},|w||g|^{q},|w f g| \in L$ and, if $p \neq 0$,

$$
0<m \leq|f(x)||g(x)|^{-q / p} \leq M, \quad \forall x \in E
$$

If $p>1$, or if $p<0$ and $A\left(|w||f|^{p}\right)+A\left(|w||g|^{q}\right)>0$, then

$$
(M-m) A\left(|w||f|^{p}\right)+\left(m M^{p}-M m^{p}\right) A\left(|w||g|^{q}\right) \leq\left(M^{p}-m^{p}\right) A(|w f g|) .
$$

This inequality is reversed if $0<p<1$ and $A\left(|w||f|^{p}\right)+A\left(|w||g|^{q}\right)>0$.

Theorem 11.2 (Diaz-Metcalf's inequality). Let $E \subset \mathbb{R}^{n}$ be as in Theorem 3.7. For $p \neq 1$, let $q=$ $p /(p-1)$. Assume $|w||f|^{p},|w||g|^{q},|w f g|$ are $\Delta$-integrable on $E$ and, if $p \neq 0$,

$$
0<m \leq|f(x)||g(x)|^{-q / p} \leq M, \quad \forall x \in E .
$$

If $p>1$, or if $p<0$ and at least one of the two integrals on the left-hand side of the following inequality is positive, then

$$
\begin{aligned}
& (M-m) \int_{E}|w(t)||f(t)|^{p} \Delta t+\left(m M^{p}-M m^{p}\right) \int_{E}|w(t)||g(t)|^{q} \Delta t \\
& \quad \leq\left(M^{p}-m^{p}\right) \int_{E}|w(t) f(t) g(t)| \Delta t .
\end{aligned}
$$

This inequality is reversed if $0<p<1$ and at least one of the two integrals on the left-hand side is positive.

Proof. Just apply Theorems 11.1 and 3.7. 
The last two results in this section follow from [2, Theorem 4.16 and Theorem 4.18] in the same way as Theorem 11.2 follows from Theorem 11.1.

Theorem 11.3. Let $E, p, q, w, f, g, m, M$ be as in Theorem 11.2. If $p>1$, then

$$
\begin{aligned}
\int_{E}|w(t) f(t) g(t)| \Delta t \geq & |p|^{1 / p}|q|^{1 / q} \frac{(M-m)^{1 / p}\left(m M^{p}-M m^{p}\right)^{1 / q}}{\left|M^{p}-m^{p}\right|} \\
& \times\left(\int_{E}|w(t)||f(t)|^{p} \Delta t\right)^{1 / p}\left(\int_{E}|w(t)||g(t)|^{q} \Delta t\right)^{1 / q} .
\end{aligned}
$$

This inequality is reversed if $p<0$ or $0<p<1$, provided at least one of the two integrals on the right-hand side is positive.

Proof. Just apply [2, Theorem 4.16] and Theorem 3.7.

Theorem 11.4. Let $E, p, q, w, f, g, m, M$ be as in Theorem 11.2 and assume

$$
0<m<F(x) \leq M, \quad 0 \leq G(x) \leq M, \quad \forall x \in E,
$$

where $F=f(f+g)^{-q / p}$ and $G=g(f+g)^{-q / p}$. Let $K(p, q, m, M)$ denote the constant on the righthand side of the inequality in Theorem 11.3. If $p>1$, then

$$
\begin{aligned}
& \left(\int_{E}|w(t)|(|f(t)|+|g(t)|)^{p} \Delta t\right)^{1 / p} \\
& \quad \geq K(p, q, m, M) \times\left\{\left(\int_{E}|w(t)||f(t)|^{p} \Delta t\right)^{1 / p}+\left(\int_{E}|w(t)||g(t)|^{p} \Delta t\right)^{1 / p}\right\} .
\end{aligned}
$$

This inequality is reversed if $0<p<1$, or if $p<0$ and the integral on the left-hand side is positive.

Proof. Just apply [2, Theorem 4.18] and Theorem 3.7.

\section{Further Converses of Jensen's Inequality}

Several results from the previous sections are sometimes also called converses of Jensen's inequality. This section is concerned with some further converses of Jensen's inequality. The five results presented follow from the specified results in [2] in the same way as Theorem 5.2 follows from Theorem 5.1.

Theorem 12.1. (a) Assume $\Phi \in C(I, \mathbb{R})$ is convex, where $I=[m, M]$ with $m<M$ such that $\Phi^{\prime \prime}(x) \geq 0$ with equality for at most isolated points of I. Assume further that either

(i) $\Phi(x)>0$ for all $x \in I$,

$\left(\mathrm{i}^{\prime}\right) \Phi(x)>0$ for all $m<x<M$ with either $\Phi(m)=0, \Phi^{\prime}(m) \neq 0$, or $\Phi(M)=0, \Phi^{\prime}(M) \neq 0$, 
(ii) $\Phi(x)<0$ for all $x \in I$,

(ii') $\Phi(x)<0$ for all $m<x<M$ with precisely one of $\Phi(m)=0, \Phi(M)=0$.

Let $E \subset \mathbb{R}^{n}$ be as in Theorem 3.7, and suppose $f$ is $\Delta$-integrable on $E$ such that $f(E)=I$. Moreover, let $h: E \rightarrow \mathbb{R}$ be $\Delta$-integrable such that $\int_{E}|h(t)| \Delta t>0$. Then

$$
\frac{\int_{E}|h(t)| \Phi(f(t)) \Delta t}{\int_{E}|h(t)| \Delta t} \leq \lambda \Phi\left(\frac{\int_{E}|h(t)| f(t) \Delta t}{\int_{E}|h(t)| \Delta t}\right)
$$

holds for some $\lambda>1$ in cases (i), ( $\left.i^{\prime}\right)$, or $\lambda \in(0,1)$ in cases (ii), (ii'). More precisely, a value of $\lambda$, depending only on $m, M, \Phi$, may be determined as follows: define $v=(\Phi(M)-\Phi(m)) /(M-m)$. If $v=0$, let $\tilde{x} \in(m, M)$ be the unique solution of the equation $\Phi^{\prime}(x)=0$; then $\lambda=\Phi(m) / \Phi(\tilde{x})$. If $v \neq 0$, let $\tilde{x} \in[m, M]$ be the unique solution of the equation $v \Phi(x)-\Phi^{\prime}(x)(\Phi(m)+v(x-m))=0$; then $\lambda=v / \Phi^{\prime}(\tilde{x})$. Moreover, one has $\tilde{x} \in(m, M)$ in the cases (i), (ii).

(b) Let all the hypotheses of (a) hold except that $\Phi$ is concave on I with $\Phi^{\prime \prime}(x) \leq 0$ with equality for at most isolated points of I. Then

$$
\frac{\int_{E}|h(t)| \Phi(f(t)) \Delta t}{\int_{E}|h(t)| \Delta t} \geq \lambda \Phi\left(\frac{\int_{E}|h(t)| f(t) \Delta t}{\int_{E}|h(t)| \Delta t}\right)
$$

where $\lambda$ is determined as in (a). Furthermore, $\lambda>1$ holds if $\Phi(x)<0$ for all $x \in(m, M)$, and $0<\lambda<1$ holds if $\Phi(x)>0$ for all $x \in(m, M)$.

Proof. Just apply [2, Theorem 3.39] and Theorem 3.8.

Theorem 12.2. (a) Let $E, f, I, m, M, h, v$ be as in Theorem 12.1, and let $\Phi \in \mathrm{C}(I, \mathbb{R})$ be differentiable such that $\Phi^{\prime}$ is strictly increasing on $I$. Then

$$
\frac{\int_{E}|h(t)| \Phi(f(t)) \Delta t}{\int_{E}|h(t)| \Delta t} \leq \lambda+\Phi\left(\frac{\int_{E}|h(t)| f(t) \Delta t}{\int_{E}|h(t)| \Delta t}\right)
$$

for $\lambda=\Phi(m)-\Phi(\tilde{x})+v(\tilde{x}-m) \in\left(0,(M-m)\left(v-\Phi^{\prime}(m)\right)\right)$, where $\tilde{x} \in(m, M)$ is the unique solution of the equation $\Phi^{\prime}(x)=v$.

(b) Let all the hypotheses of (a) hold except that $\Phi^{\prime}$ is strictly decreasing on I. Then

$$
\Phi\left(\frac{\int_{E}|h(t)| f(t) \Delta t}{\int_{E}|h(t)| \Delta t}\right) \leq \curlywedge+\frac{\int_{E}|h(t)| \Phi(f(t)) \Delta t}{\int_{E}|h(t)| \Delta t}
$$

for $\lambda=\Phi(\tilde{x})-\Phi(m)-v(\tilde{x}-m) \in\left(0,(M-m)\left(\Phi^{\prime}(m)-v\right)\right)$ with $\tilde{x}$ given in $(a)$.

Proof. Just apply [2, Theorem 3.41] and Theorem 3.8. 
Theorem 12.3. In addition to the assumptions of Theorem 5.2, let $J \subset \mathbb{R}$ be an interval such that $J \supset \Phi(I)$ and assume that $F: J \times J \rightarrow \mathbb{R}$ is increasing in the first variable. Then

$$
\begin{aligned}
& F\left(\frac{\int_{E}|h(t)| \Phi(f(t)) \Delta t}{\int_{E}|h(t)| \Delta t}, \Phi\left(\frac{\int_{E}|h(t)| f(t) \Delta t}{\int_{E}|h(t)| \Delta t}\right)\right) \\
& \quad \leq \max _{x \in[m, M]} F\left(\frac{M-x}{M-m} \Phi(m)+\frac{x-m}{M-m} \Phi(M), \Phi(x)\right) \\
& \quad=\max _{\theta \in[0,1]} F(\theta \Phi(m)+(1-\theta) \Phi(M), \Phi(\theta m+(1-\theta) M)),
\end{aligned}
$$

and the right-hand side of the inequality is an increasing function of $M$ and a decreasing function of $m$.

Proof. Just apply [2, Theorem 3.42] and Theorem 3.8.

Theorem 12.4. Under the same hypotheses as in Theorem 12.3 except that $F$ is decreasing in its first variable, one has

$$
\begin{gathered}
F\left(\frac{\int_{E}|h(t)| \Phi(f(t)) \Delta t}{\int_{E}|h(t)| \Delta t}, \Phi\left(\frac{\int_{E}|h(t)| f(t) \Delta t}{\int_{E}|h(t)| \Delta t}\right)\right) \\
\quad \geq \min _{x \in[m, M]} F\left(\frac{M-x}{M-m} \Phi(m)+\frac{x-m}{M-m} \Phi(M), \Phi(x)\right) \\
\quad=\min _{\theta \in[0,1]} F(\theta \Phi(m)+(1-\theta) \Phi(M), \Phi(\theta m+(1-\theta) M)),
\end{gathered}
$$

and the right-hand side of the inequality is a decreasing function of $M$ and an increasing function of $m$.

Proof. Just apply [2, Theorem 3.42'] and Theorem 3.8.

Theorem 12.5. Assume $\Phi \in C(I, \mathbb{R})$ is convex, where $I \subset \mathbb{R}$ is an interval. Let $E \subset \mathbb{R}^{n}$ be as in Theorem 3.7. Let $h: E \rightarrow \mathbb{R}$ be $\Delta$-integrable on $E$ such that $0<\int_{E}|h(t)| \Delta t<\alpha$ for some $\alpha \in \mathbb{R}$. If $|h| f$ and $|h|(\Phi \circ f)$ are $\Delta$-integrable on $E$ and $a \in I$ is such that

$$
\frac{\alpha a-\int_{E}|h(t)| f(t) \Delta t}{\alpha-\int_{E}|h(t)| \Delta t} \in I
$$

then

$$
\Phi\left(\frac{\alpha a-\int_{E}|h(t)| f(t) \Delta t}{\alpha-\int_{E}|h(t)| \Delta t}\right) \geq \frac{\alpha \Phi(a)-\int_{E}|h(t)| \Phi(f(t)) \Delta t}{\alpha-\int_{E}|h(t)| \Delta t}
$$

Proof. Just apply [2, Lemma 4.25] and Theorem 3.7. 


\section{Acknowledgment}

The research of the J. Pečarić is supported by the Croatian Ministry of Science, Education and Sports under the Research Grants 117-1170889-0888.

\section{References}

[1] M. Bohner and A. Peterson, Dynamic Equations on Time Scales: An Introduction with Application, Birkhäuser, Boston, Mass, USA, 2001.

[2] J. E. Pečarić, F. Proschan, and Y. L. Tong, Convex Functions, Partial Orderings, and Statistical Applications, vol. 187 of Mathematics in Science and Engineering, Academic Press, Boston, Mass, USA, 1992.

[3] J. L. W. V. Jensen, "Sur les fonctions convexes et les inégalités entre les valeurs moyennes," Acta Mathematica, vol. 30, no. 1, pp. 175-193, 1906.

[4] R. Agarwal, M. Bohner, and A. Peterson, "Inequalities on time scales: a survey," Mathematical Inequalities and Applications, vol. 4, no. 4, pp. 535-557, 2001.

[5] F.-H. Wong, C.-C. Yeh, and W.-C. Lian, "An extension of Jensen's inequality on time scales," Advances in Dynamical Systems and Applications, vol. 1, no. 1, pp. 113-120, 2006.

[6] U. M. Özkan, M. Z. Sarikaya, and H. Yildirim, "Extensions of certain integral inequalities on time scales," Applied Mathematics Letters, vol. 21, no. 10, pp. 993-1000, 2008.

[7] Q. Sheng, M. Fadag, J. Henderson, and J. M. Davis, "An exploration of combined dynamic derivatives on time scales and their applications," Nonlinear Analysis: Real World Applications, vol. 7, no. 3, pp. 395-413, 2006.

[8] M. R. S. Ammi, R. A. C. Ferreira, and D. F. M. Torres, "Diamond- $\alpha$ Jensen's inequality on time scales," Journal of Inequalities and Applications, Article ID 576876, 13 pages, 2008.

[9] M. Bohner and G. Sh. Guseinov, "Multiple integration on time scales," Dynamic Systems and Applications, vol. 14, no. 3-4, pp. 579-606, 2005.

[10] M. Bohner and G. Sh. Guseinov, "Multiple Lebesgue integration on time scales," Advances in Difference Equations, vol. 2006, Article ID 26391, 13 pages, 2006.

[11] B. Jessen, "Bemærkninger om konvekse Funktioner og Uligheder imellem Middelværdier. I," Matematisk Tidsskrift B, vol. 2, pp. 17-28, 1931.

[12] P. R. Beesack and J. E. Pečarić, "On Jessen's inequality for convex functions," Journal of Mathematical Analysis and Applications, vol. 110, no. 2, pp. 536-552, 1985.

[13] C. Dinu, "Hermite-Hadamard inequality on time scales," Journal of Inequalities and Applications, Article ID 287947, 24 pages, 2008.

[14] D. R. Anderson, "Time-scale integral inequalities," Journal of Inequalities in Pure and Applied Mathematics, vol. 6, no. 3, article 66, 15 pages, 2005.

[15] C. Dinu, "A weighted Hermite Hadamard inequality for Steffensen-Popoviciu and HermiteHadamard weights on time scales," Analele Stiintifice ale Universitatii Ovidius Constanta, vol. 17, no. 1, pp. 77-90, 2009.

[16] J. E. Pečarić and P. R. Beesack, “On Jessen's inequality for convex functions. II,” Journal of Mathematical Analysis and Applications, vol. 118, no. 1, pp. 125-144, 1986.

[17] R. A. C. Ferreira, M. R. S. Ammi, and D. F. M. Torres, "Diamond-alpha integral inequalities on time scales," International Journal of Mathematics and Statistics, vol. 5, no. A09, pp. 52-59, 2009.

[18] M. R. S. Ammi and D. F. M. Torres, "Hölder's and Hardy's two dimensional diamond-alpha inequalities on time scales," Annals of the University of Craiova. Mathematics and Computer Science Series, vol. 37, no. 1, pp. 1-11, 2010.

[19] M. Dresher, "Moment spaces and inequalities," Duke Mathematical Journal, vol. 20, pp. 261-271, 1953.

[20] J. Aczél, "Some general methods in the theory of functional equations in one variable. New applications of functional equations," Uspekhi Matematicheskikh Nauk, vol. 11, no. 3(69), pp. 3-68, 1956. 


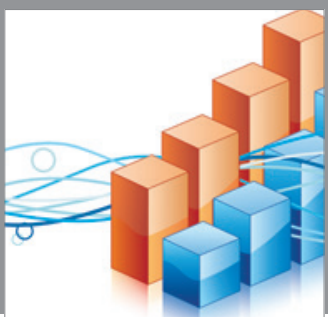

Advances in

Operations Research

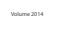

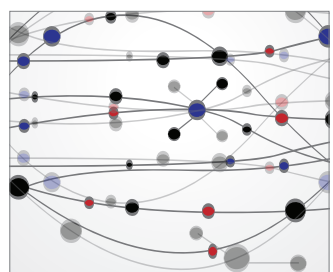

\section{The Scientific} World Journal
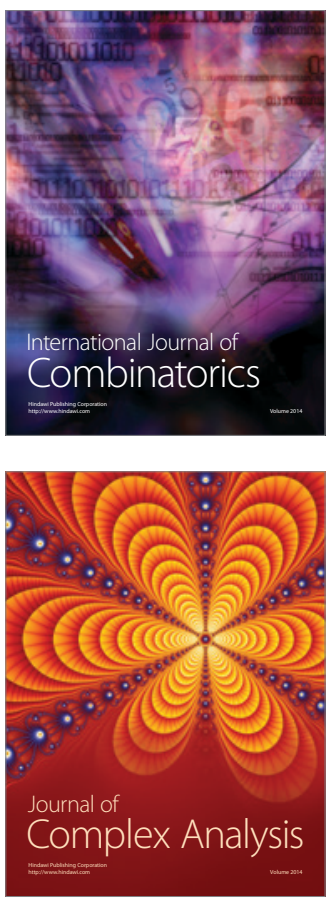

International Journal of

Mathematics and

Mathematical

Sciences
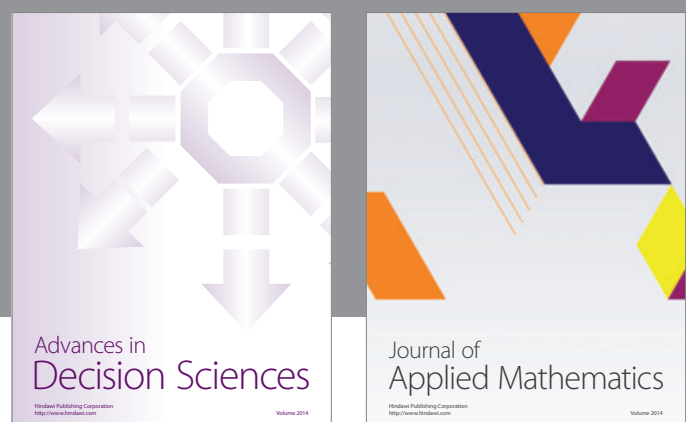

Journal of

Applied Mathematics
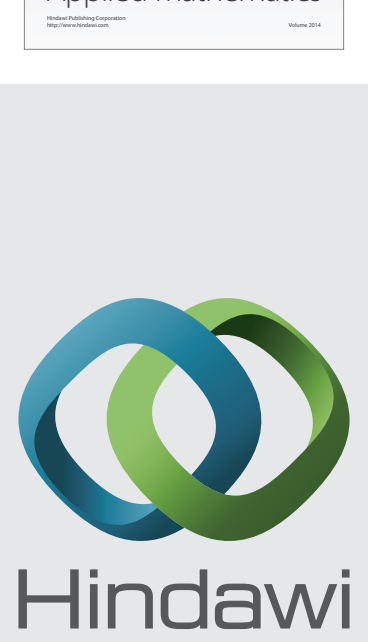

Submit your manuscripts at http://www.hindawi.com
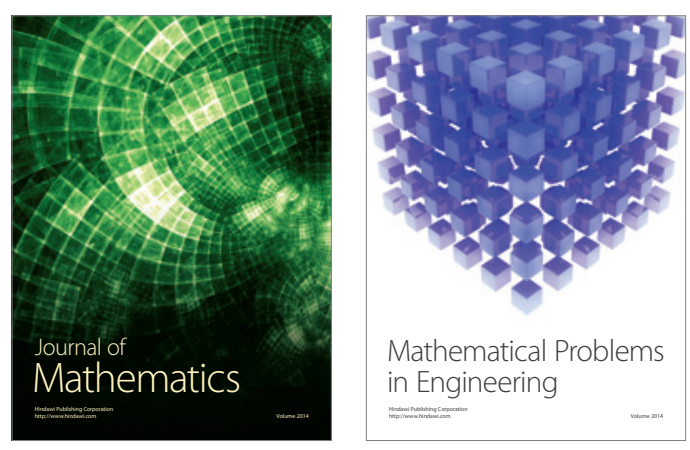

Mathematical Problems in Engineering
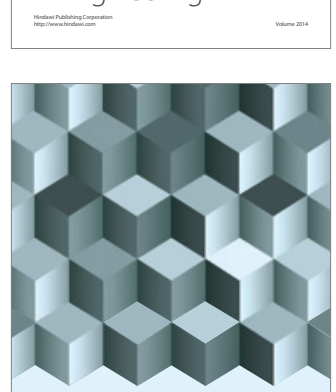

Journal of

Function Spaces
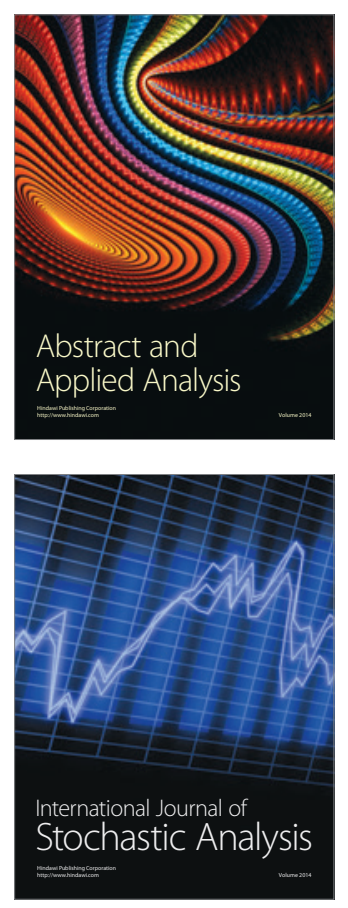

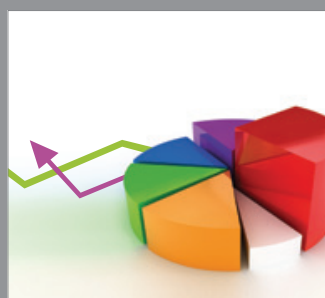

ournal of

Probability and Statistics

Promensencen
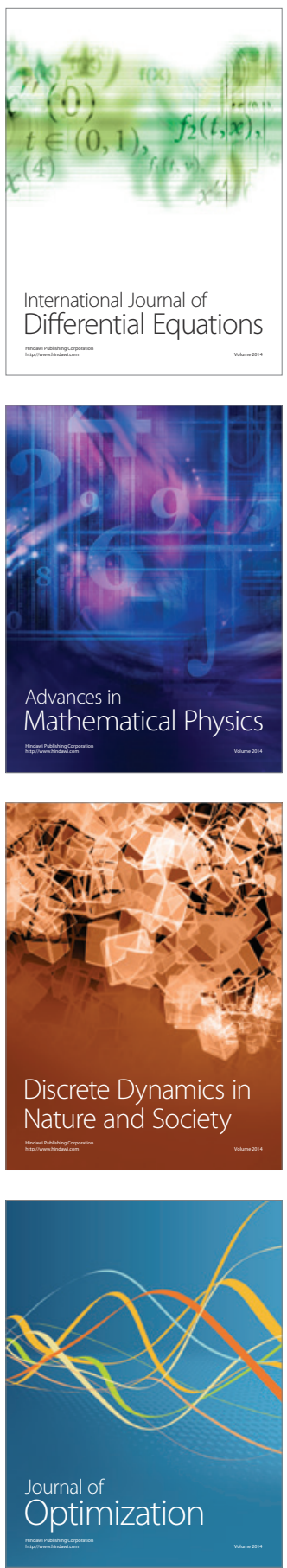